\title{
ORT_27 - Phenotypic and functional characterization of innate immunity cells in the establishment of murine pulmonary malaria
}

Marcos Vinicius Rangel Ferreira ${ }^{1 *}$; Lucas Freire Antunes ${ }^{1}$; Carina Heusner Gonçalves ${ }^{1}$; Uyla Ornellas Garcia $^{1}$; Mônica Lucas Ribeiro de Almeida ${ }^{1}$; Cláudio T Daniel-Ribeiro ${ }^{1}$; Patricia M Martins ${ }^{1}$; Flávia Lima Ribeiro Gomes ${ }^{1}$.

${ }^{1}$ Fiocruz/IOC.

Introduction: Malaria is an infectious-parasitic disease caused by a protozoa of the genus Plasmodium, and is one of the main public health problems worldwide. The parasitosis may progress to severe forms with pulmonary complications associated with acute respiratory distress syndrome (MA-ARDS). Infection with $P$. falciparum, $P$. vivax, $P$. ovale or $P$. knowlesi can lead to severe respiratory symptoms of MAARDS in humans. In murine models of malaria, MA-SDRA is characterized by increased permeability of the pulmonary microvasculature endothelium and inflammation. However, little is known about the immunoregulatory mechanisms associated with the pathogenesis of MA-ARDS.

Objective: The aim of this study was to investigate the kinetics of lung innate immune cell recruitment and the polarization profile of alveolar macrophages. C57BL/6 and Balb/c mice were infected with $1 \times 10^{6}$ erythrocytes parasitized with Plasmodium berghei ANKA.

Methodology: On days 4 and 6 after infection, the animals were euthanized and the bronchoalveolar lavage (BAL) and tissue digestion was performed to obtain the samples and analyzes. Subpopulations of myeloid cells (inflammatory monocytes, alveolar macrophages, neutrophils and /or eosinophils) present in BAL and lung tissue were analyzed by flow cytometry and nitric oxide, arginase and cytokines were measured in the BAL supernatant.

Results: C57BL/6 infected-mice showed pulmonary dysfunction and edema as attested by increased organ weight and protein content in the BAL and pulmonary interstitium. In contrast, BALB/c mice showed a small increase in the organ weight and total proteins in the BAL, alterations that did not interfere with lung function. We observed differences in the percentage and/or total number of inflammatory monocytes and neutrophils, in both compartments, in BALB/c and C57BL/6 mice, throughout the infection. Parasitized mice of both strains, although exhibiting different kinetics, showed reduction in the percentage and total number of alveolar macrophages, throughout the disease. Interestingly, alveolar macrophages of C57BL/6 mice exhibited higher expression of CD206 and MHC class II as well as reduction in the percentage of positive cells for iNOS enzyme. In addition, an increase in arginase and nitric oxide enzyme activities was detected in the BAL of infected C57BL/6 mice. No alteration of eosinophil population was noted throughout the infection in both mouse strains. Analysis of the cytokine profile revealed a significant increase in the ratio between the levels of IFN- $\gamma$ / IL-10 and TNF-a / IL-10 in the BAL of C57BL/6 infected-mice.

Conclusion: Our findings show that C57BL/6 and BALB/c mice exhibit different dynamics of cell population in the lung and plasticity of alveolar macrophages in the BAL, phenomena that may be associated with the development or not of the pulmonary complications observed in C57BL/6 and BALB/c, respectively. This study brings findings about innate immunity cells in development of pulmonary malaria and contributes to the future development of more effective therapy.

Keywords: Alveolar macrophage; Plasmodium; Inflammation 